Anesthetic.-The choice of an anesthetic is more often determined by the idiosyncrasy of the operator than the necessity of the case. There were 13 operations made with cocain infiltration. Of these 3 were completed with chloroform. While there are occasional cases in which local anesthesia may be necessary, we have noticed but little difference in the shock or general condition of the cases from the anesthetic employed. We now use ether anesthesia, preceded twenty minutes by hypodermic of $1 / 6 \mathrm{gr}$. of morphin and 1/120 gr. atropin. The table is placed in an elevated slanting position with the head up (reverse Trendelenburg). The tumor is rendered prominent by a roll beneath the neck if it does not interfere with respiration.

Operation.-We prefer the collar or transverse incision. In tumors of medium size this crosses the center of the tumor, in larger tumors it crosses the upper third and the lower flap is split vertically to the sternum if necessary.

The incision is through skin and platysma muscle, the flap being raised to expose freely the muscles covering the gland, namely, the sternohyoid and thyroid, the inner portions of the sternomastoid and omohyoid. In medium-sized growths muscle separation will permit of the delivery of the tumor. The muscle section of the sternohyoid and thyroid group, if made, should be near their upper attachment so as not to interfere with their nerve supply and also to break the line of scar formation trom that part of the skin. After the removal of the gland, the severed muscles are carefully united by suture. The upper section also permits of early ligation of the superior thyroid artery which is the key to the situation.

The more firm and rounded tumors, with a strong capsule, can be readily enucleated after incising the capsule and penetrating the gland tissue to the cyst. We usually loosen the gland from the outside of the capsule first. On viewing the tumor, the true capsule has the luster and appearance of peritoneum. If one is not sure, incise between the vessels. Should hemorrhage be severe, lifting up the tumor by its capsule will reduce the bleeding until sutures can be placed for its control. In parenchymatous and colloid tumors we make extirpation of one lobe and the isthmus. After exposing it the upper pole is elevated and the superior thyroid artery is cut between the ligatures. The lateral veins are clamped and cut, the lower pole is elevated into the wound, incision is made along the outer posterior border, and the capsule is brushed off with gauze to the median line. As a rule, the inferior thyroid is now ligated close to the tumor as the capsule is brushed back. This leaves the nerve behind and usually out of sight. The isthmus is clamped and closed by suture. If the enucleation method for ademoma and cysts leaves a badly torn lobe it can be removed. Sometimes extirpation of one lobe is made and enucleation of cystic masses is done on the opposite side, after the method of Porta, Billroth and Socin. The resection method of Mikulicz was only employed in one case, but with good result. The ligation of the thyroid arteries as a method was not practiced. We avoid rough handling of the portion of gland to be left.

Should there be a large area of cut gland exposed it is burned over with carbolic acid, followed by alcohol, or often Harrington's No. 9 solution is applied over the cut tissues to close the lymph absorbents and favor drainage. If there has been but little traumatism we do not drain. Large incisions and large cavities we drain temporarily. All exophthalmic cases are drained as freely as the most septic wound.

These cases absorb some wound secretion containing. colloid, causing rise in temperature and increase of pulse the next day. To delay absorption, especially in exophthalmic goiter, patients are given large quantities of salines by the bowel directly after the operation and for the first two to four days. If not retained by the bowel, they are given subcutaneous administration.

The wound is closed by subcuticular suture, great pains being taken to unite the platysma to prevent spreading of the scar.

\section{OPERATION FOR THE SHORTENING OF THE BROAD LIGAMENTS.}

\section{AN ANATOMIC AND CLINICAL STUDY.* \\ ARTHUR E. HERTZLER, M.D., PH.D. KANSAS CITY, MO.}

The material underlying this study was as follows: Forty adults and a larger number of pelves of newborn infants were dissected. Microscopic sections were made in various planes of the broad ligaments. Serial sections were made of these structures in fetuses of from three to six months, and also of pelvis of younger

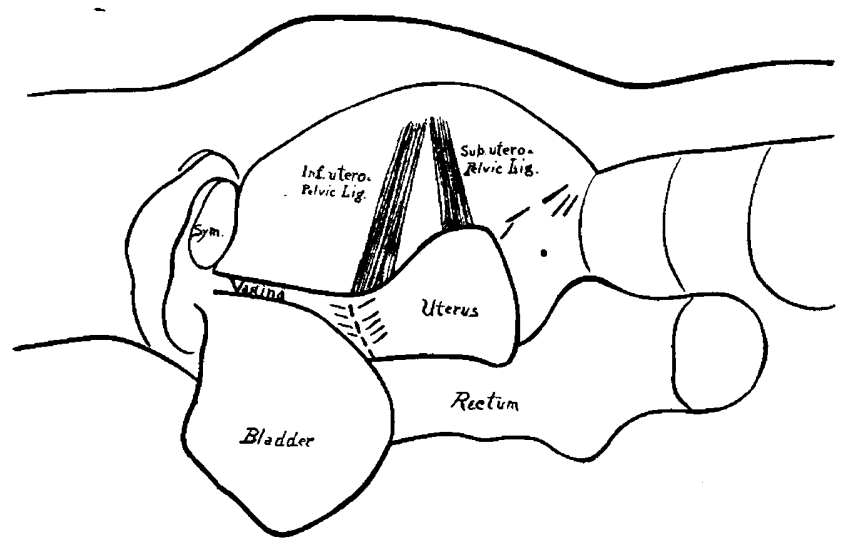

Figure 1.

embryos. The whole clinical study is based on 46 cases operated on in the past four years.

Works on anatomy are in accord in describing the broad ligament as formed of two layers of peritoneum. It is described in a recent work by my distinguished teacher, Waldeyer, ${ }^{1}$ in a general way as composed of two serous layers, between which are vessels and nerves, many smooth muscle fibers and more or less connective tissue.

This generally accepted opinion becomes questionable when it is considered that the peritoneum, strictly speaking, can be dissected from the pelvis complete, except from the fundus of the uterus, leaving the ligaments intact. This furnishes indisputable evidence that the accepted description of the broad ligaments is inadequate, and that they are true ligaments.

In corroborating this statement the dissector must have a clear notion as to what is comprised by the peritoneum. It is incorrect to regard all the fibers below the endothelial layer of cells as comprising the peritoneum. I have in course of preparation an extensive

* This article was received in July, 1906

* Read hefore the South Kansas Medical Soclety, April 18, 1906. 1. "Das Becken," p. 530. 
exposition of this subject and a detailed discussion can not be entered into at this time. It may be anticipated, however, that I regard the peritoneum as composed of a layer of endothelial cells, an elastic layer and a thin layer of looser connective tissue. The actual thinness of the peritoneum can be appreciated by picking up a mesentery on the finger and noting the delicacy of the double thickness so presented. When this is kept in mind the statement that the peritoneum acts as a suspensory ligament nowhere under any condition will not seem extravagant. The peritoneum covers all the intraabdominal suspensory ligaments but is nowhere identical with them. This is particularly true of the broad ligament, which is the strongest of them all.

When the broad ligaments are exposed, by the removal of the peritoneum, two bands are seen extending lateralward from the uterus. The upper one, arising from the cornu of the uterus, has its upper border immediately below the origin of the mesosalpinx. Its lower border extends from 5 to $15 \mathrm{~mm}$. below the ovarian artery. The fibrous bands are arranged about this vessel for the greater part of its extent across from the pelvic brim. The lower ligament begins at the point of approach of the uterine artery to the uterus. A few

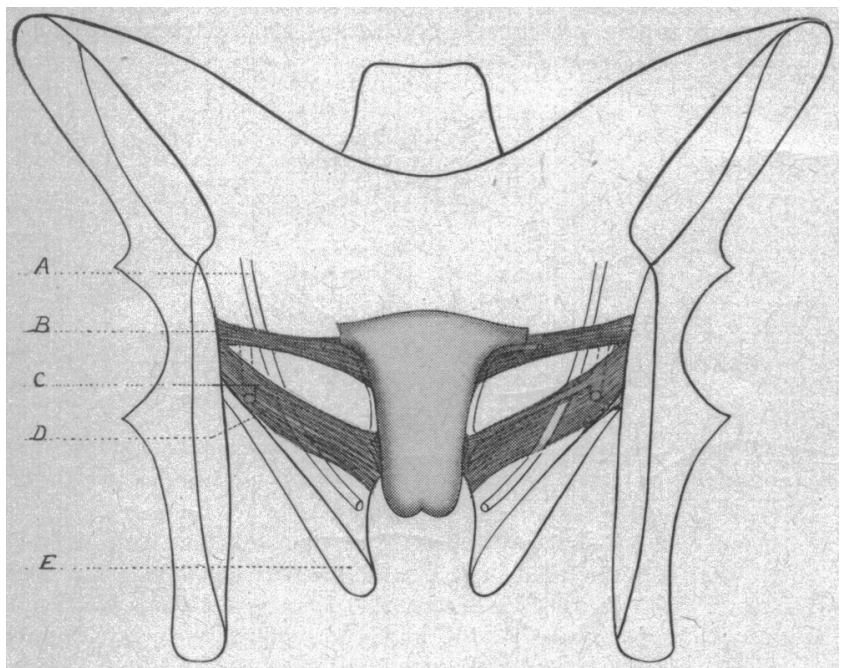

Fig. 2.-A. Ureter. B. Superior uteropelvic ligament. C. Uterine artery. D. Inferior uteropelvic ligament. E, Levator ani.

fibers pass above this vessel, as also above the ureter, but by far the greater part is below. At one time in the development no fibers are to be found above the artery and ureters but as early as the fourth month a few fibers pass above it. Below the entrance of the artery the ligament forms dense masses of fibers and continues to become more dense until the insertion of the vagina into the cervix is reached. At this point of insertion fibers unite with the upper portion of the vagina. These are not of great importance and are of relatively late appearance developmentally. The unimportance of these fibers, as well as those passing above the uterine artery, can best be appreciated by the ease with which blunt dissection from these structures can be made on the operating table.

The upper ligament extends directly lateralward from its point of origin to meet the wall of the pelvis at the white line or just above it. The lower band extends lateralward and upward, from the point of origin already described, to meet the pelvic wall immediately below the upper band. The fibers of the two bands intermingle at their attachment to the pelvic fascia. Some of the fibers pass upward above the pelvic brim to become continuous with the retro-peritoneal fascia. The extent of the insertion varies in the different subjects dissected. Some experience is required to demonstrate them with facility. In subjects hardened in strong formalin no difficulty should be experienced. In fresh subjects the dissector is likely to lose a portion of the fibers in the removal of the peritoneum if care is not exercised. Figure 1, which is a faithful reproduction of a specimen preserved in my laboratory, gives a good idea of the size of these structures.

It would be more in accord with the usages of descriptive anatomy to describe the ligaments as arising at the white line and having their insertion in the cornu and utero-cervical junction respectively, but in dissection it is more convenient to begin the dissection at the uterine end of the ligaments. If it is desired to designate these structures by a separate name, that of superior and inferior utero-pelvic ligaments is suggested. Or, if it is desired to have names in harmony with the B. N. A.,

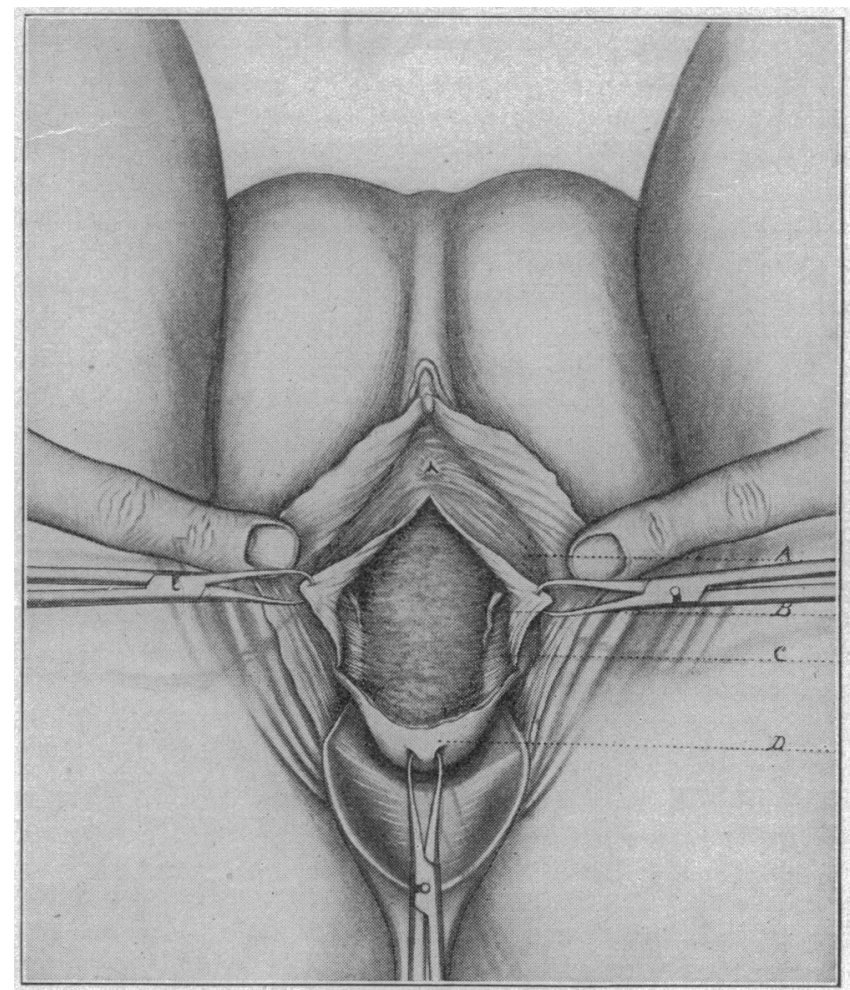

Fig. 3.-A, Ureter. B. Uterine artery. C. Inferior utero pelvic ligament. D. Cervix.

ligamentum utero-pelvicum superior and lig. utero-pelvicum inferior might be appropriate.

Microscopically these ligaments are composed of dense bands of fibrous tissue with an interlacement of smooth muscular fibers. The loose areolar tissue is situated, for the most part, subperitoneally and above the lower ligament. This statement can be best appreciated by the study of fetal pelves.

The relation of the uterine ligaments to the uterus, as they concern us in a surgical way, may be understood by reference to Figure 2. It will be obserred how the uterus is anchored by these structures and what the effect on the position of the uterus is when they are elongated, and conversely the effect on the descensus when the ligaments are shortened.

Figure 3 shows the ligaments as exposed by vaginal incision. They can be readily dissected out with the end of the finger and freed from the uterine artery and 
from the ureter if desired; the latter, however, is not necessary. When loosened in this way the ligaments can be drawn out from 3 to $6 \mathrm{~cm}$., depending on the degree of prolapse, without difficulty. Figure 4 shows the ligaments drawn out and attached.

To the experienced vaginal operator, familiar with pelvic anatomy, little more need be said. I have hardly done the operation exactly twice alike. The later operations have, however, been fairly uniform. A transverse incision is made in front of the cervix and the end of the ligament exposed by passing the finger between it and the vagina, thus getting below and behind it. It is then easy to follow it up as far as the uterine artery. With care the peritoneum can be lifted up without injury. In the earlier operations the artery was tied if the uterus was heavy and the patient near the menopause. If it is desired to do so the vessel can be tied extraperitoneally. After the exposure of the ligaments they are severed between two ligatures. The first ligature is passed as closely as possible to the cervix, the other $1 \mathrm{~cm}$. or less lateralward. If the uterine artery

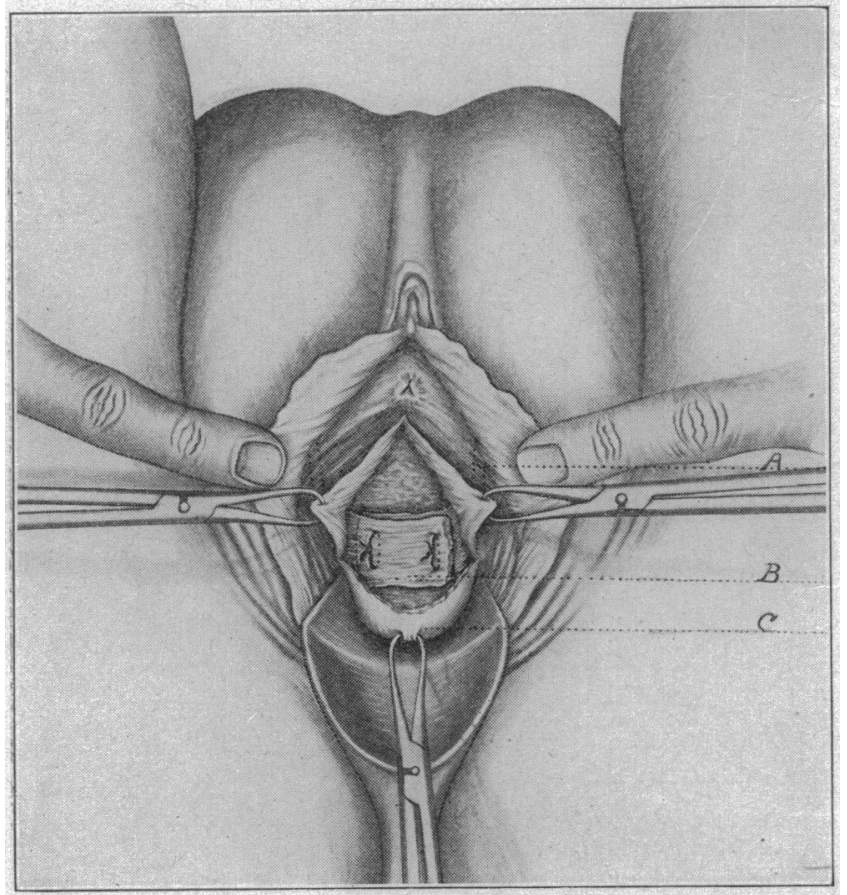

Fig. 4.-A. Ureter. B. Inferior uteropelvic ligament overlapping C. Cervix.

is tied this precaution is unnecessary. After free exposure of the ligaments they are overlapped as far as may be necessary to raise the cervix high enough. The method of overlapping is unimportant. An imitation of the method used by Mayo in umbilical hernia is satisfactory and easily applied. Pyoctannin gut is used for all sutures, plain gut for ligatures.

I have made use of this operation in all cases of prolapse. In cases of prolapse of high degree either a Dührssen fixation or a Freund-Wertheim has been added.

In patients in the child-bearing age I have added to this shortening of the uterine ligaments the shortening of the round ligaments to hold the fundus forward. This is done by a method believed to have some points of superiority. It is briefly as follows: A Wertheim incision is made over the pubes and the abdomen is opened according to his method. The pelvic contents are examined and gizen the needed treatment. The round ligaments are reached by retracting the lateral angles of the wound thus exposing the external inguinal ring. A curved forceps is passed along the inguinal canal and passed into the abdomen and the ligament siezed subperitoneally and dragged along the inguinal canal into the external ring. The grasp of the ligament within the abdomen is guided by the index finger of the left hand within the abdomen. By this method the peritoneum over the round ligament is not opened and there are no extraneous hands to cause trouble and only preexisting openings are utilized. If the uterus is fixed by adhesions this operation must precede the shortening of the uterine ligaments, otherwise it is done afterward. As a matter of fact fixed uteri are usually not prolapsed, thus do not require a shortening of the uterine ligaments. The operative results have been satisfactory. I do not offer the operation as a finished product but I believe the operation is mechanically more correct than those now employed. As to the correctness of the anatomic observation I feel confident.

\section{A CASE OF CHRONIC OSTEO-ATROPHIC ARTHRITIS.*}

R. S. LAVENSON, M.D.

Assistant Pathologist to the Hospital of the University of Pennsylvania, and to the Philadelphia General Hospltal Assoctate In the William Pepper Laboratory of Clinical Medicine; Visiting Physician to the Fhiladelphia Home for Incurables. PHILADELPHIA.

The extraordinary features presented by the bones and joints of the patient forming the subject of this communication justify, in my opinion, the publication of the case as an almost unique one.

Patient.-K. I., a woman aged 54, white, single, and a native of Ireland, is an inmate of the Philadelphia Home for Incurables.

Family History.-Her family history presents no features bearing any relation to her own condition. There is no history of rheumatism.

Previous Medical Condition.-The patient's previous history shows her to have been strong and healthy excepting for some undeterminable infection when she was about 10 years old. Her work was confined to household duties. At the time of the onset of the present trouble she was a woman of about 5 feet 3 inches in height, weighing in the neighborhood of 135 pounds.

Present Illness.-About twenty-seven years ago the patient noted some pain and stiffness in the right hip, coming on after a severe wetting. This disappeared within a few weeks, and for a year she was entirely well. At this time pain and stiffness occurred in the third toe of the right foot. Very shortly after, the right knee was similarly involved, and then successively, the right hand and wrist and the left wrist. Later, most of the remaining joints of the body were involved, and within a few months from the onset of symptoms in the toe, practically all the joints of the body were affected. There was some swelling, but pain on movement and stiffness were the most prominent features. The patient does not recall having had any fever at this time. About twenty years ago, approximately six years after the onset, the patient noted that the thumb of the right hand was becoming shorter, and soon after observed a similar process in all fingers of both hands. There was a gradual progression of this shortening until a few years ago, since which time she believes there has been no increase in the process.

Physical Examination.-The patient is an extremely small woman, sitting continuously in a chair with the trunle flexed at about a right angle to the thighs and the head slightly

* From the Willam Pepper Laboratory of Clinical Mudicine Phoebe A. Heirst Foundation. 